\title{
Emerging personalized oncology: sequencing and systems strategies
}

“...these techniques provide unprecedented deep insights into the cancer genome structure and function, shaping the future of personalized oncology."

\author{
William Cho', Demosthenes E Ziogas ${ }^{2,3}$, Christos Katsios ${ }^{2,3}$ \& Dimitrios H \\ Roukos $^{\star 2,3}$ \\ 'Department of Clinical Oncology, Queen Elizabeth Hospital, Hong Kong, China \\ 2Department of Surgery, University Hospital of loannina, loannina, Greece \\ ${ }^{3}$ Biosystems \& Synthetic Genomic Network Medicine Center, loannina University, loannina, Greece \\ *Author for correspondence: Tel.: +30 2651007423 = Fax: +30 2651007094 = droukos@uoi.gr
}

Genome-wide mapping technologies have revolutionized biomedical sciences. The increasing validity of next-generation sequencing (NGS) technologies and the freefalling costs of human genome sequencing, revealing causal differences in DNA and RNA sequences between health and diseases $[1,2]$, now raise the question of implementing the clinical genome into medical practice. Particularly, in cancer, applying NGS and array-based high-throughput techniques in both primary tumors and metastatic biopsies for a comprehensive genome, epigenome and transcriptome analysis provides fascinating clinical implications [3]. These potential implementations include a novel mutation-based taxonomy [4], personalized cancer diagnostics [5,6] and tailored targeted treatment decisions [7]. However, there is still a plethora of challenges, such as validity, ethics and interpretation of structural and functional genome data. Many technological and scientific hurdles must be surpassed in order to understand the whole-genome architecture and functional regulatory networks driving cell behavior in health and cancer before reaching clinical personalized oncology. Can these problems be overcome in order to improve survival or even cure by targeting the cancer genome of an individual patient?

The Human Genome Project, completed in 2003, required 13 years and US $\$ 3$ billion to provide the first draft of a complete human genome sequence. This project marked a revolution in biomedical sciences. Since then, the technological advances have been so rapid that the cost of whole-genome sequencing (WGS) is currently only $\$ 5000$. By the end of 2012, the $\mathrm{NIH}$ will have funded whole-exome sequencing (WES) or WGS of samples from approximately 70,000 subjects involved in research protocols. The advent of $\$ 1000$ genome sequencing will have a profound effect on biomedical science by effectively democratizing genome-scale sequencing for research and clinical purposes [8]. Each human genome is unique and the first WGS or WES studies published revealed a wide heterogeneity in the cancer genome sequence among patients with the same organspecific and tumor-node-metastasis stage $[5,7]$. Over the next few years, with the completion of tissue-based WGS/WES from thousands of cancer patients by the International Cancer Genome Consortium [9] and other projects, a causal mutation-based landscape will emerge for each cancer type, promising the development of new taxonomy-based personalized genetic characterization and treatment of cancer [4].

\section{Personalized strategies}

During the past two decades, efforts for more effective management of cancer with survival prolongation and fewer adverse effects, by using an individualized approach, have already been translated into medical practice. Clinicians have previously recognized that patients with preneoplastic lesions or small early-stage gastrointestinal tumors can be safely treated with endoscopic mucosal or submucosal resection with improved quality of life and excellent long-term survival, preventing surgery. In the mid-1990s, the discovery that heritable mutations in $B R C A 1$ or $B R C A 2$ cause hereditary breast or ovarian cancer in $70-80 \%$ of women carrying these mutations led to the current genetic testing-based clinical decision-making on primary prevention with surgery or intensive surveillance. However, most patients are diagnosed in advanced disease, while BRCA1/2 breast cancer cases account for fewer than $10 \%$ of annually diagnosed breast cancer patients. During the past decade, biomarkers and singlepathway inhibitors have been rapidly evolved and are in wide clinical use for the treatment of patients with advanced cancer.

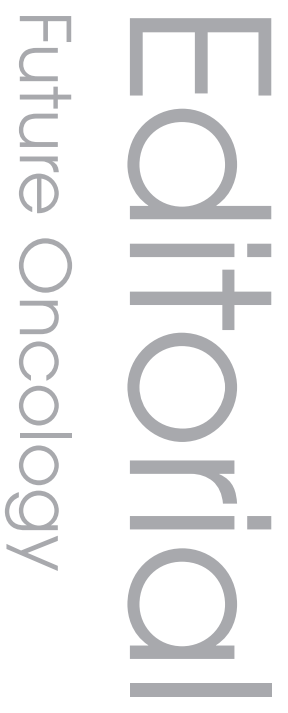

\section{Keywords}

- biomarker = cancer genome - next-generation sequencing - personalized oncology

- sequencing = transcriptome

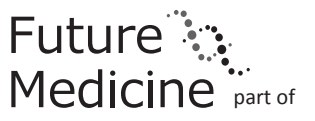


From chemotherapy to targeted therapy Surgery for complete tumor resection has been evolved and standardized to provide locoregional control for most solid tumors. Systemic chemotherapy in the adjuvant preoperative or postoperative setting, or the metastatic setting, has also been standardized, improving the oncological outcomes of millions of patients. However, a high proportion of patients relapse and die from the disease. Despite its therapeutic benefit, chemotherapy is not only associated with toxic adverse effects, but also, in some cases, may contribute to recurrence and disease progression. Indeed, the results of a more recent WGS study using NGS in patients with acute myeloid leukemia provide scientific evidence that, although most primary tumor cells are sensitive and are killed by the initial chemotherapy, a subclone of the founding tumor cell clone survives, gains additional mutations and is expanded at relapse. DNA-damaging chemotherapy itself contributes to this treatment failure [5]. To increase response and survival rates, and to reduce relapses and adverse effects, tumor-targeted treatment is of key interest in biomedical research.

\section{Evolution \& limitations}

The pharmaceutical and biomolecular diagnostic industries are currently shifted towards signaling transduction-inhibitor drugs development and validation. Crucial cellular processes, including survival, growth, differentiation, proliferation and apoptosis, are regulated by signal transduction from the cell surface to the nucleus. Deregulation or amplification of this signal transmission by the accumulation of genetic and epigenetic changes, and, ultimately, gene expression dysfunction, collectively result in major diseases such as cancer, among others. Inhibiting or restoring deregulated signaling pathways and gene expression patterns in cancer cells is the key principle in the effort to develop effective signal transduction drugs. The US FDA has approved 33 monoclonal antibodies and small-molecule tyrosine kinase inhibitors for metastatic cancer, as well as one for clinical use in the adjuvant setting, with many others in clinical development [101].

Trastuzumab and vemurafenib (PLX4032) represent two targeted cancer drugs that have achieved clinical success. Evidence suggests that the anti-HER2 humanized monoclonal antibody trastuzumab improves overall survival in HER2positive metastatic breast and gastric cancer, and is the only targeted drug approved in the adjuvant setting among all solid tumors, specifically for the treatment of early-stage breast cancer [10] The tyrosine kinase inhibitor vemurafenib, for patients with metastatic melanoma and $B R A F$ V600E mutations, has been demonstrated to significantly improve overall survival [11].

However, even among patients selected on the basis of HER2 amplification or BRAF mutation status, intrinsic or acquired resistance remains a daunting challenge. For the vast majority of other targeted drugs, the results from Phase III randomized trials are less promising. Either a progression-free survival improvement alone, without any overall survival benefit or no treatment response has been reported $[12,101]$. For example, two Phase III randomized trials by Baselga and colleagues, published in 2012 in the New England Journal of Medicine, for metastatic breast cancer demonstrated no overall survival benefit $[13,14]$. More specifically, by adding the mTOR inhibitor everolimus to endocrine therapy in hormone receptor-positive patients [13], or pertuzumab (another anti-HER2 monoclonal antibody) to trastuzumab and chemotherapy in metastatic HER2-positive patients [14], the progression-free survival benefit should be weighed against the side effects of these drugs as described by Baselga et al. [13,14]. Out of approximately 150 kinase-targeted drugs that are in clinical development and many more that are in various stages of preclinical development, most are used for oncological indications. Collectively, evidence from current Phase III trials limits the expectations of these drugs, with either temporary or no antitumor effects and no overall survival benefit [12].

\section{Revolutionary sequencing \& mapping strategies}

Sequencing (WGS/WES) tumor biopsies from individual patients provides an unprecedented identification of causal mutations underlying each patient's tumor. This powerful information, along with the low-cost promises for a '\$1000 genome' in 2013, raise questions regarding the clinical implementation of the mutation landscape-based concept for personalized diagnostics and targeted therapeutics. Although a recent validation study comparing WGS by NGS machines from Illumina, Inc. (CA, USA) and Complete Genomics, Inc. (CA, USA) revealed a high sensitivity, specificity and concordance with single-nucleotide variants, insertions and deletions, there were some differences between the two platforms. The authors proposed that a dual WGS from the two platforms would reach a very high accuracy in assessing point mutations [15]. 


\section{Beginning the epoch of clinical genomics}

In 2012, several large-scale pilot studies using NGS for sequencing the genomes of patients have been launched, for example, in Norway [102] and at least two US universities, such as the Baylor College of Medicine in Houston (TX, USA) and the University of California, Los Angeles (CA, USA) [103]. Advanced translational bioinformatics have evolved to provide multiscale databases of all classes of mutations - point mutations, copy-number variants and genomic rearrangements - for most cancer types [16].

\section{Challenges in translating genome sequencing into the clinic}

By contrast with the research world, clinicians need genomic WGS/WES tests with proven clinical utility in Phase III trials. However, no Phase III trials based on genomic WGS/WES tests yet exist and many challenges with respect to NGS data storage and interpretation, as well as regulatory, ethical and financial problems, need to be overcome.

\footnotetext{
"Sequencing the primary tumor and

relapse genomes by next-generation

sequencing provides an unprecedented capacity for understanding the metastatic potential of some tumors."
}

Recently, Roychowdhury et al. extracted both the DNA and RNA from samples of two patients with metastatic solid cancer and applied the 'sequence everything' approach for NGS-based WGS, WES and RNA sequencing for transcriptome as well as gene expression mapping [7]. This sequencing strategy allows the assessment of all classes of genetic variants, including point mutations, copy-number alterations and genomic rearrangements, as well as gene amplification and overexpression. Roychowdhury et al. suggest that this comprehensive mutational landscape of individual patients' samples by integrative highthroughput sequencing facilitates clinical trials for drug development that target individual patients' tumor-activating signaling pathways and thus reach personalized oncology [7].

Sequencing the primary tumor and relapse genomes by NGS provides an unprecedented capacity for understanding the metastatic potential of some tumors. Ding et al. provide information on the clonal evolution of mutations in acute myeloid leukemia complementary to recently described mutational evolution in breast and pancreatic cancer metastases [5]. Taken together, these clonal evolution data on breast and pancreatic metastatic cancer [17-19], and acute myeloid leukemia [5], suggest that some mutations and DNA-damaging chemotherapy may contribute to clonal selection and initial therapeutic resistance.

Chromatin immunoprecipitation followed by high-throughput sequencing provides a fascinating technology for transcriptome mapping at a genome-wide level. As the estrogen receptor (ER) is the defining transcription factor of breast cancer and its target genes orchestrate growth and the endocrine response, ER-binding events could be used to assess therapeutic response and prognosis. Shifting from cell line models to clinical samples, Ross-Innes et al. were the first to use chromatin immunoprecipitation followed by high-throughput sequencing to map genome-wide ER-binding events, in frozen primary breast cancer samples from patients with both good and poor clinical outcomes, and in distant ER-positive metastases [6]. They found that unique ER-binding regions in primary tumors were associated with relapse and poor outcome. By contrast with the report by Ding et al. in acute myeloid leukemia [5] and another breast cancer report on clonal evolution [17], Ross-Innes et al. found that the differential ER-binding program observed in tumors from patients with a poor outcome was not due to the selection of a rare subpopulation of cells, but was due to FOXA1-mediated reprogramming of ER binding in a rapid timescale [6]. Therefore, this chromatin immunoprecipitation followed by high-throughput sequencing-based transcription factor mapping in primary tumor material provides the potential to discover novel biomarkers for predicting therapeutic response and outcome in individual patients.

\section{The future of personalized oncology}

The three most recent genomic studies represent excellent paradigms on applying NGS techniques to cancer tissues and blood samples and how combining these experimental omics data with patients' clinical data could lead to translational genomic medicine [5-7]. However, these studies either provide diverse results on the molecular mechanisms of recurrence, which underly therapeutic resistance $[5,6]$, or raise questions regarding the efficacy of the proposed selection of mutation-targeting signaling pathway inhibitors [7], given that this project does not consider the dynamics or complexity of the physical and functional network of intracellular signal transduction interactions and cell-cell connectivity [20]. 


\section{Signaling pathway networks}

Why should emphasis and priority be given to innovative research for understanding the highly complex network of cellular signal transduction interactions? First, experimental evidence now reveals that cell and organ function is orchestrated by dynamic molecular networks and cell-cell functional connectivity $[2,20,21]$. In cancer, deregulation of interacting biological systems, including transcriptional activity, noncoding RNAs and the epigenome, ultimately lead to chromatin state and gene expression pattern changes [22]. Second, evidence from current Phase III randomized trials reveals a modest efficacy with respect to overall survival improvement of most of the single signaling pathway inhibitors currently available [12-14,101].

In the near future, progress will occur in the understanding of DNA-binding proteins at the transcriptome level; noncoding RNAs, including miRNAs, and protein-protein interactions at interactome level; and epigenome modifications and their interactions. Collectively, all these physical and functional interaction networks, also known as interactome, synthesize a highly dynamic spatiotemporal molecular network [2,20-26]. In cancer, targeting both this deregulated differential molecular network and

genome structural causal variants can result in the development of highly effective drugs that are tailored to individual patients in order to improve overall survival and cure rates.

\section{Conclusion}

Beyond the clinical success of trastuzumab, emerging research emphasis is focused on how to develop novel robust biomarkers for improving the tailoring of available targeted drugs and on how to develop the next generation of inhibitors of the network of signaling pathway interactions. Although the time has not yet arrived for wide clinical implementation of NGS and other omics technologies, these techniques provide unprecedented deep insights into the cancer genome structure and function, shaping the future of personalized oncology.

\section{Financial \& competing interests disclosure \\ The authors have no relevant affiliations or financial involvement with any organization or entity with a financial interest in or financial conflict with the sub- ject matter or materials discussed in the manuscript. This includes employment, consultancies, honoraria, stock ownership or options, expert testimony, grants or patents received or pending, or royalties. \\ No writing assistance was utilized in the production of this manuscript.}

\section{References}

1. Green ED, Guyer MS; National Human Genome Research Institute. Charting a course for genomic medicine from base pairs to bedside. Nature 470 (7333), 204-213 (2011).

2. Guttman M, Rinn JL. Modular regulatory principles of large non-coding RNAs. Nature 482(7385), 339-346 (2012).

3. Mirnezami R, Nicholson J, Darzi A. Preparing for precision medicine. N. Engl. J. Med. 366(6), 489-491 (2012).

4. Stratton MR. Exploring the genomes of cancer cells: progress and promise. Science 331(6024), 1553-1558 (2011).

5. Ding L, Ley TJ, Larson DE et al. Clonal evolution in relapsed acute myeloid leukaemia revealed by whole-genome sequencing. Nature 481(7382), 506-510 (2012).

6. Ross-Innes CS, Stark R, Teschendorff AE et al. Differential oestrogen receptor binding is associated with clinical outcome in breast cancer. Nature 481(7381), 389-393 (2012).

7. Roychowdhury S, Iyer MK, Robinson DR et al. Personalized oncology through integrative high-throughput sequencing: a pilot study. Sci. Transl Med. 3(111), 111ra121 (2011).
8. Phimister EG, Feero WG, Guttmacher AE. Realizing genomic medicine. N. Engl. J. Med. 366(8), 757-759 (2012).

9. Hudson TJ, Anderson W, Artez A et al.; International Cancer Genome Consortium. International network of cancer genome projects. Nature 464(7291), 993-998 (2010).

10. Boekhout AH, Beijnen JH, Schellens JH. Trastuzumab. Oncologist 16(6), 800-810 (2011).

11. Chapman PB, Hauschild A, Robert C et al. Improved survival with vemurafenib in melanoma with BRAF V600E mutation. N. Engl. J. Med. 364(26), 2507-2516 (2011).

12. Fabbro D, Cowan-Jacob SW, Möbitz H, Martiny-Baron G. Targeting cancer with small-molecular-weight kinase inhibitors. Methods Mol. Biol. 795, 1-34 (2012).

13. Baselga J, Campone M, Piccart M. Everolimus in postmenopausal hormone-receptor-positive advanced breast cancer. $N$. Engl. J. Med. 366(6), 520-529 (2012).

14. Baselga J, Cortés J, Kim SB et al. Pertuzumab plus trastuzumab plus docetaxel for metastatic breast cancer. N. Engl. J. Med. 366(2), 109-119 (2012).

15. Lam HY, Clark MJ, Chen R et al. Performance comparison of whole-genome sequencing platforms. Nat. Biotechnol. 30(1), 78-82 (2011).
16. Wang MD. In the spotlight: bioinformatics, computational biology and systems biology. IEEE Rev. Biomed. Eng. 4, 3-5 (2011).

17. Ding L, Ellis MJ, Li S et al. Genome remodelling in a basal-like breast cancer metastasis and xenograft. Nature 464 , 999-1005 (2010).

18. Yachida S, Jones S, Bozic I et al. Distant metastasis occurs late during the genetic evolution of pancreatic cancer. Nature 467, 1114-1117 (2010).

19. Navin N, Kendall J, Troge J et al. Tumour evolution inferred by single-cell sequencing. Nature 472, 90-94 (2011).

20. Ideker T, Krogan NJ. Differential network biology. Mol. Syst. Biol. 8, 565 (2012).

21. Baker DJ, Wijshake T, Tchkonia T et al. Clearance of p16Ink4a-positive senescent cells delays ageing-associated disorders. Nature 479(7372), 232-236 (2011).

22. Baylin SB, Jones PA. A decade of exploring the cancer epigenome - biological and translational implications. Nat. Rev. Cancer 11(10), 726-734 (2011).

23. Roukos DH, Katsios C, Liakakos T. Genotype-phenotype map and molecular networks: a promising solution in overcoming 
colorectal cancer resistance to targeted treatment. Expert Rev. Mol. Diagn. 10(5), 541-545 (2010).

24. Katsios C, Roukos DH. Individual genomes and personalized medicine: life diversity and complexity. Pr. Med. 7(4), 347-350 (2010).

25. Roukos DH. Complete genome sequencing and network modeling to overcome trastuzumab resistance. Pharmacogenomics 11(8), 1039-1043 (2010).
26. Roukos DH. Next-generation, genome sequencing-based biomarkers: concerns and challenges for medical practice. Biomark. Med. 4(4), 583-586 (2010).

\section{Websites}

101. NIH. Targeted Cancer Therapies. www.cancer.gov/cancertopics/factsheet/ Therapy/targeted

(Accessed 17 May 2012)
102. Nature. Norway to bring cancer-gene tests to the clinic.

www.nature.com/news/norway-to-bringcancer-gene-tests-to-the-clinic-1.9949 (Accessed 17 May 2012)

103. Nature. Sequencing set to alter clinical landscape.

www.nature.com/news/sequencing-set-toalter-clinical-landscape-1.10032

(Accessed 17 May 2012) 